\title{
ANNALES
}

UNIVERSITATIS MARIAE CURIE-SKŁODOWSKA

LUBLIN - POLONIA

VOL. LXV, NO. 2, 2011

SECTIO A

$53-61$

\author{
REINER KÜHNAU
}

\section{Eine Klasse nichtschlichter konformer Abbildungen mit einer schlichten quasikonformen Fortsetzung. II}

\author{
Im Gedenken an Professor dr. Jan Krzyż
}

\begin{abstract}
We study a dual analogue of the class $\Sigma(\kappa)$ of hydrodynamically normalized schlicht conformal mappings $g(z)$ of the exterior of the unit circle with a $\frac{1+\kappa}{1-\kappa}$-quasiconformal extension, namely now those (non-schlicht) mappings $g(z)$ for which $\overline{g(z)}$ has such a quasiconformal extension.
\end{abstract}

1. Einleitung. Wir betrachten in Fortsetzung von [8] stetige Abbildungen, auch nichtschlichte, der vollen komplexen $z$-Ebene.

1. Es sei $\Sigma(\kappa)$ die Klasse aller Abbildungen $w=g(z)$ des Äußeren $|z|>1$ des Einheitskreises mit hydrodynamischer Normierung

$$
g(z)=z+\frac{b_{1}}{z}+\frac{b_{2}}{z^{2}}+\ldots,
$$

die stetig und quasikonform nach $|z| \leq 1$ fortsetzbar sind, wobei für $|z|<1$ mit einer Konstanten $\kappa, 0 \leq \kappa<1$, gilt

$$
\left|g_{\bar{z}}\right| \leq \kappa\left|g_{z}\right|
$$

d.h., $g(z)$ erfülle eine Beltramigleichung mit einem Beltramikoeffizienten $\nu(z)$, für den $|\nu(z)| \leq \kappa<1$ und die hierbei üblichen Voraussetzungen

2000 Mathematics Subject Classification. Primary: 30C55; Secondary: 30C62, 30C50.

Key words and phrases. Non-schlicht functions, quasiconformal extension. 
gelten. (Bekanntlich sind diese Abbildungen $g(z)$ von selbst stets schlicht in der ganzen $z$-Ebene; $\Sigma(0)$ enthält nur die Identität.) Natürlich gilt

$$
\Sigma\left(\kappa_{1}\right) \subset \Sigma\left(\kappa_{2}\right) \text { für } \kappa_{1}<\kappa_{2} .
$$

2. Es sei $\Sigma(1-)$ die Gesamtheit der Grenzfunktionen, die für $|z|>1$ bei gleichmäßiger Konvergenz von Funktionen aus $\bigcup_{0<\kappa<1} \Sigma(\kappa)$ entstehen (d.h., bei gleichmäßiger Konvergenz der jeweiligen Funktionen $g(z)-z$ für $|z| \geq r$ bei jedem festen $r>1)$. Bekanntlich ist $\Sigma(1-)$ mit der Abbildungsklasse $\Sigma$ identisch. Das sind die nur für $|z|>1$ betrachteten schlichten konformen Abbildungen der Form (1). (Alle Abbildungen aus $\Sigma(1-)$ gehören nämlich zu $\Sigma$, und jede Abbildung $g(z)$ aus $\Sigma$ läßt sich durch Abbildungen aus $\bigcup \Sigma(\kappa)$ beliebig approximieren, indem man $\frac{1}{r} g(r z)$ mit $r>1$ und $r \rightarrow 1$ betrachtet.) Natürlich ist $\bigcup_{0 \leq \kappa<1} \Sigma(\kappa)$ eine (echte) Teilmenge von $\Sigma=\Sigma(1-)$.

3. Es sei $\widetilde{\Sigma}(\kappa)$ die Klasse aller analytischen Abbildungen $g(z)$ des Äußeren $|z|>1$ des Einheitskreises mit hydrodynamischer Normierung (1), die stetig nach $|z| \leq 1$ fortsetzbar sind, wobei für $|z|<1$ mit der Konstanten $\kappa>1$ gilt

$$
\left|g_{\bar{z}}\right| \geq \kappa\left|g_{z}\right|
$$

d.h., $\overline{g(z)}$ erfülle eine Beltramigleichung mit einem Beltramikoeffizienten $\nu(z)$, für den $|\nu(z)| \leq \frac{1}{\kappa}<1$ und die üblichen Voraussetzungen gelten. Die Abbildungen $g(z)$ sind zwangsläufig nicht schlicht in der ganzen $z$-Ebene. Beim Bilde von $|z|=1$ erfolgt - anschaulich gesprochen - ein "Umkrempeln". Wir betrachten in dieser Mitteilung bei $\kappa>1$ vornehmlich die Teilklasse $\widetilde{\Sigma_{s}}(\kappa)$. Das seien diejenigen Abbildungen $g(z)$ aus $\widetilde{\Sigma}(\kappa)$, für die $|z|<1$ durch $\overline{g(z)}$ schlicht und $\frac{\kappa+1}{\kappa-1}$-quasikonform (und also orientierungserhaltend) abgebildet wird. Als Beispiel vergleiche man (6).

Wir haben hier natürlich

$$
\widetilde{\Sigma}\left(\kappa_{1}\right) \supset \widetilde{\Sigma}\left(\kappa_{2}\right) \text { und } \widetilde{\Sigma_{s}}\left(\kappa_{1}\right) \supset \widetilde{\Sigma_{s}}\left(\kappa_{2}\right) \text { für } \kappa_{1}<\kappa_{2} .
$$

Die Klasse $\widetilde{\Sigma}(\infty)$ wäre sinngemäß aufzufassen als Klasse der Funktionen $g(z)$, die für alle $z$ stetig sind, für $|z|>1$ analytisch bis auf den einfachen Pol in $z=\infty$ mit hydrodynamischer Normierung (1), während $\overline{g(z)}$ für $|z|<1$ analytisch ist. Diese Klasse ist leer, da die für $|z|>1$ analytische Funktion $\overline{g(1 / \bar{z})}$ längs $|z|=1$ mit $g(z)$ übereinstimmen müßte, also auch für $|z|>1$, was nicht angeht wegen des Poles in $z=\infty$.

4. Es sei $\widetilde{\Sigma}(1+)$ die Gesamtheit der Grenzfunktionen, die für $|z|>1$ bei gleichmäßiger Konvergenz von Funktionen aus $\bigcup_{1<\kappa} \widetilde{\Sigma}(\kappa)$ entstehen.

Die Klasse $\widetilde{\Sigma}(\kappa)$ bzw. $\widetilde{\Sigma_{s}}(\kappa)$ erscheint in vieler Hinsicht dual zur Klasse $\Sigma(\kappa)$. Für die Abbildungen (1) der Klasse $\Sigma(\kappa)$ gilt der sogenannte 
Flächensatz [7], [10], [11]

$$
\sum_{k=1}^{\infty} k\left|b_{k}\right|^{2} \leq \kappa^{2} \quad(0<\kappa<1) .
$$

Es gibt verschiedene hierzu verwandte Flächensätze, auch für mehrfach zusammenhängende Gebiete: [1-6].

Für die Abbildungen der Klasse $\widetilde{\Sigma_{s}}(\kappa)$ gilt dagegen

$$
\sum_{k=1}^{\infty} k\left|b_{k}\right|^{2} \geq \kappa^{2} \quad(1<\kappa) .
$$

Das Gleichheitszeichen steht genau für die Abbildungen (6) (mit $p=\kappa$ ). Ungleichung (5) wurde in [8] für die Klasse $\widetilde{\Sigma_{s}}(\kappa)$ bemerkt (in [2], [3] für mehrfach zusammenhängende Gebiete verallgemeinert). Man kann (5) für die größere Klasse $\widetilde{\Sigma}(\kappa)$ verallgemeinern und hierzu den an [10] gelehnten Beweis übertragen. Allerdings kann dann die unendliche Reihe in (5) divergieren, so daß (5) gegenstandslos wird. Das wurde in [4] bemerkt. (Dieses Phänomen der möglichen Divergenz der Reihe in (5) kann in der Teilklasse $\widetilde{\Sigma_{s}}(\kappa)$ nicht auftreten wegen des Zusammenhanges mit einem Flächeninhalt.)

Unten werden wir u.a. in Ergänzung von (5) allgemeine Koeffizienten-Ungleichungen vom Grunskyschen Typ für die Klasse $\widetilde{\Sigma_{s}}(\kappa)$ herleiten.

Ein entscheidender Unterschied zwischen den Klassen $\Sigma(\kappa)$ und $\widetilde{\Sigma}(\kappa)$ besteht im Folgenden. Bei Vorgabe eines Beltrami-Koeffizienten $\nu(z)$ mit $|\nu(z)| \leq \kappa<1$ existiert bekanntlich immer genau eine zugehörige Abbildung $g(z) \in \Sigma(\kappa)$. Das Analoge für die Klasse $\widetilde{\Sigma}(\kappa)$ ist nicht immer richtig; vgl. [9]. Eine einfache Charakterisierung der Beltrami-Koeffizienten $\nu(z)$, für die ein $g(z) \in \widetilde{\Sigma}(\kappa)$ oder gar ein $g(z) \in \widetilde{\Sigma_{s}}(\kappa)$ existiert, so daß $\overline{g(z)}$ diesen Beltrami-Koeffizienten besitzt, steht noch aus. Immerhin gibt es zu diesem Problem eine äquivalente funktionentheoretische Randwertaufgabe [9].

2. $\widetilde{\Sigma}(1+)$ enthält die Klasse $\boldsymbol{\Sigma}$. Diese Aussage der Überschrift ist ein gewisses Gegenstück zur oben bemerkten Aussage $\Sigma=\Sigma(1-)$.

Wir beweisen sogar den

Satz 1. Jede Abbildung $g(z)$ der Klasse $\Sigma$ läßt sich beliebig durch Abbildungen aus $\bigcup_{1<\kappa} \widetilde{\Sigma_{s}}(\kappa)$ (nur für $|z|>1$ betrachtet) approximieren (wieder im Sinne gleichmäßiger Konvergenz).

Beweis. Zunächst gilt dies bei denjenigen Abbildungen $g(z) \in \Sigma$, bei denen aus $|z|=1$ ein abgeschlossener analytischer Schlitz entsteht. Denn solche Abbildungen lassen sich analytisch fortsetzen nach $|z|>r$ mit einem $r<1$, wobei auch der Ring $r \leq|z|<1$, insbesondere $|z|=r$, schlicht abgebildet 
wird. In die Kreisscheibe $|z| \leq r$ läßt sich demnach noch eine schlichte quasikonforme Abbildung als stetige Fortsetzung "einspannen", so daß wir die gewünschten Approximationen in der Gestalt $\frac{1}{r} g(r z)$ erhalten, mit anschließendem Grenzübergang $r \rightarrow 1$.

Hat man nun eine beliebige Abbildung $g(z)$ der Klasse $\Sigma$, so läßt sich diese bekanntlich durch solche Schlitzabbildungen der betrachteten Art beliebig approximieren, eine ähnliche Prozedur wie bei der ursprünglichen Behandlung der Theorie der Löwnerschen Differentialgleichung. Als analytische Schlitze fungieren z.B. die Bilder von Kreisbögen $|z|=1+\epsilon,|\arg z|<$ $2 \pi-\epsilon$ ( $\epsilon$ klein $)$.

3. Beispiel. Zur Illustrierung dieser Klassen betrachtet man als Beispiel zweckmäßig die Abbildung

$$
g(z)= \begin{cases}z+p e^{2 i \theta} \frac{1}{z} & \text { für }|z| \geq 1 \\ z+p e^{2 i \theta} \bar{z} & \text { für }|z| \leq 1 .\end{cases}
$$

Bei $0<p<1$ gilt $g(z) \in \Sigma(\kappa)$ für alle $\kappa$ mit $p \leq \kappa<1$. Bei $p>1$ ist $g(z) \in \widetilde{\Sigma}(\kappa)$, sogar $g(z) \in \widetilde{\Sigma_{s}}(\kappa)$, für alle $\kappa$ mit $1<\kappa \leq p$. Bei $p=1$ liefert $g(z)$ eine Abbildung, die in $\Sigma(1-)$ und $\widetilde{\Sigma}(1+)$ liegt.

Man übersieht klar die Abhängigkeit der Abbildungen vom Parameter $p$. Wenn $p$ von 0 bis 1 variiert, nimmt bei den schlichten Abbildungen $g(z)$ die (konstante) Dilatation in $|z|<1$ zu und strebt nach $\infty$. Sie sinkt dann nach dem Wert $p=1$ bis auf den Wert 1 (der im Grenzfalle $p \rightarrow \infty$ wieder Konformität entspräche). Also: Bei $p>1$ kommt die Abbildung für $|z|<1$ für $p \rightarrow \infty$ der (indirekten) Konformität immer näher. Ab $p=1$ erfolgt ein "Umkrempeln" längs des Bildes von $|z|=1$. Für $p>1$ liegt beim Bilde von $|z|=1$ eine Falte (Kante) vor. Oder: Das Bild der $z$-Ebene ist im Sinne von P. Koebe ein Faltflächenstück (jedenfalls keine Riemannsche Fläche). Diese einfache Konfiguration hat man allerdings nicht immer bei den Abbildungen der Klasse $\widetilde{\Sigma}(\kappa)$ - vgl. [9].

Ein Beispiel einer Funktion $g(z) \in \widetilde{\Sigma}(\kappa)$, die nicht zu $\widetilde{\Sigma_{s}}(\kappa)$ gehört, findet man unten.

4. Koeffizientenbedingungen vom Grunskyschen Typ in der Klasse $\widetilde{\boldsymbol{\Sigma}_{\boldsymbol{s}}}(\boldsymbol{\kappa})$. Wir folgen hier den Überlegungen (und weitgehend den Bezeichnungen) in [11], S. 56 ff. und S 290/291, mit geeigneten Modifizierungen.

Sei also $g(z)$ eine Abbildung von $\widetilde{\Sigma_{s}}(\kappa)$, wie in (1) normiert. Da nur die Entwicklung um $\zeta=\infty$ benutzt wird, können auch wieder wie in der Klasse $\Sigma$ bzw $\Sigma(\kappa)$ die Faberschen Polynome $\phi_{n}(w)$ definiert werden durch

$$
\log \frac{g(\zeta)-w}{\zeta}=-\sum_{n=1}^{\infty} \frac{1}{n} \phi_{n}(w) \zeta^{-n} \text { für } w \in \mathbb{C},|\zeta| \text { groß. }
$$


Die Grunsky-Koeffizienten $b_{k l}$ werden wieder definiert durch

$$
\log \frac{g(z)-g(\zeta)}{z-\zeta}=-\sum_{k, l=1}^{\infty} b_{k l} z^{-k} \zeta^{-l},|z|,|\zeta| \text { groß },
$$

wobei diese Entwicklung freilich nicht im ganzen Äußeren des Einheitskreises möglich ist, da $g(z)$ dort nicht schlicht ist. Aus (7) folgt andererseits

$$
\log \frac{g(z)-g(\zeta)}{z-\zeta}=-\sum_{k=1}^{\infty} \frac{1}{k}\left[\phi_{k}(g(z))-z^{k}\right] \zeta^{-k},
$$

so daß durch Vergleich mit (8) fließt

$$
\phi_{k}(g(z))=z^{k}+k \sum_{l=1}^{\infty} b_{k l} z^{-l}
$$

zunächst für große $|z|$, dann aber allgemein für $|z|>1$, da dort die linke Seite analytisch ist für $z \neq \infty$.

Setzen wir nun mit komplexen Parametern $\lambda_{k}$ das Polynom

$$
h(w)=\sum_{k=1}^{m} \frac{\lambda_{k}}{k} \phi_{k}(w)
$$

an, ergibt sich

$$
h(g(z))=\sum_{k=1}^{m} \frac{\lambda_{k}}{k} z^{k}+\sum_{k=1}^{\infty} d_{k} z^{-k}, \quad d_{k}=\sum_{l=1}^{m} b_{k l} \lambda_{l}, \quad \text { für }|z|>1 .
$$

Es wird weiter wie in [11], S.60, bei Integration über den positiv orientierten Einheitskreis $|z|=1$, bei Betrachtung der Funktion $h(g(z))$ im Äußeren des Einheitskreises,

$$
\frac{1}{2 \pi i} \int \bar{h} d h=\sum_{k=1}^{m} \frac{\left|\lambda_{k}\right|^{2}}{k}-\sum_{k=1}^{\infty} k\left|d_{k}\right|^{2} .
$$

Andererseits ergibt sich bei Betrachtung der Funktion $h(g(z))$ im Inneren des Einheitskreises

$$
\frac{1}{2 \pi i} \int \bar{h} d h=\frac{1}{\pi} \iint_{|z|<1}\left(\left|h_{z}\right|^{2}-\left|h_{\bar{z}}\right|^{2}\right) d x d y .
$$

Mit

$$
s(z)=\mathfrak{R e} h(g(z))=\frac{1}{2}(h+\bar{h})
$$

errechnet man wegen $\kappa\left|h_{z}\right| \leq\left|h_{\bar{z}}\right|$ für $|z|<1$

$$
4\left|s_{z}\right|^{2}=\left|h_{z}+\bar{h}_{z}\right|^{2} \leq\left(\left|h_{z}\right|+\mid h_{\bar{z}}\right)^{2} \leq \frac{\kappa+1}{\kappa-1}\left(\left|h_{\bar{z}}\right|^{2}-\left|h_{z}\right|^{2}\right),
$$


damit nach (14)

$$
\frac{1}{2 \pi i} \int \bar{h} d h \leq-\frac{4}{\pi} \frac{\kappa-1}{\kappa+1} \iint_{|z|<1}\left|s_{z}\right|^{2} d x d y
$$

(Man beachte, daß wir jetzt hier gegenüber dem klassischen Falle [11] das andere Vorzeichen bekommen.) Nun betrachten wir noch wie in [11] für $|z|<1$ die Funktion

$$
\begin{aligned}
\psi(z) & =h\left(g\left(\frac{1}{z}\right)\right)-\sum_{k=1}^{m} \frac{\lambda_{k}}{k} z^{-k}+\sum_{k=1}^{m} \frac{\overline{\lambda_{k}}}{k} z^{k} \\
& =\sum_{k=1}^{m}\left(d_{k}+\frac{\overline{\lambda_{k}}}{k}\right) z^{k}+\sum_{k=m+1}^{\infty} d_{k} z^{k},
\end{aligned}
$$

wobei $u(z)=\mathfrak{R e} \psi(\bar{z})$ für $|z|<1$ harmonisch ist und auf $|z|=1$ gleiche Randwerte wie $s(z)$ besitzt. Das Dirichletsche Prinzip (vgl. [11], S. 289) liefert also

$$
\begin{aligned}
4 \iint_{|z|<1}\left|s_{z}\right|^{2} d x d y & \geq 4 \iint_{|z|<1}\left|u_{z}\right|^{2} d x d y=\iint_{|z|<1}\left|\psi^{\prime}(z)\right|^{2} d x d y \\
& =\pi \sum_{k=1}^{m} k\left|d_{k}+\frac{\overline{\lambda_{k}}}{k}\right|^{2}+\pi \sum_{k=m+1}^{\infty} k\left|d_{k}\right|^{2} \\
& =\pi \sum_{k=1}^{\infty} k\left|d_{k}\right|^{2}+2 \pi \mathfrak{R e} \sum_{k=1}^{m} d_{k} \lambda_{k}+\pi \sum_{k=1}^{m} \frac{\left|\lambda_{k}\right|^{2}}{k} .
\end{aligned}
$$

Daneben zeitigen (13) und (17)

$$
4 \iint_{|z|<1}\left|s_{z}\right|^{2} d x d y \leq \pi \frac{\kappa+1}{\kappa-1}\left(\sum_{k=1}^{\infty} k\left|d_{k}\right|^{2}-\sum_{k=1}^{m} \frac{\left|\lambda_{k}\right|^{2}}{k}\right),
$$

dies und (19) schließlich

$$
(\kappa-1) \mathfrak{R e} \sum_{k=1}^{m} d_{k} \lambda_{k}+\kappa \sum_{k=1}^{m} \frac{\left|\lambda_{k}\right|^{2}}{k} \leq \sum_{k=1}^{\infty} k\left|d_{k}\right|^{2} .
$$

Somit gilt, da die $\lambda_{k}$ beliebig wählbar sind, der folgende Satz.

Satz 2. Für die Abbildungen $g(z)$ der Klasse $\widetilde{\Sigma_{s}}(\kappa)$ gilt für alle Systeme komplexer Zahlen $\lambda_{k}$ für die Grunsky-Koeffizienten $b_{k l}$

$$
(\kappa-1)\left|\sum_{k, l=1}^{m} b_{k l} \lambda_{k} \lambda_{l}\right|+\kappa \sum_{k=1}^{m} \frac{\left|\lambda_{k}\right|^{2}}{k} \leq \sum_{k=1}^{\infty} k\left|\sum_{l=1}^{m} b_{k l} \lambda_{l}\right|^{2} .
$$


Die bei $m=1, \lambda_{1}=1, \lambda_{k}=0$ für $k \geq 2$, entstehende Ungleichung

$$
(\kappa-1)\left|b_{1}\right|+\kappa \leq \sum_{k=1}^{\infty} k\left|b_{k}\right|^{2}
$$

liefert gegenüber dem Flächensatz (5) nichts Neues (analog wie bei $\Sigma(\kappa)$ ), nämlich sowohl nicht für $\left|b_{1}\right| \leq \kappa$ (da dann $\left.(\kappa-1)\left|b_{1}\right|+\kappa \leq \kappa^{2}\right)$, als auch nicht für $\left|b_{1}\right| \geq \kappa$, da dann

$$
(\kappa-1)\left|b_{1}\right|+\kappa \leq\left|b_{1}\right|^{2} \leq \sum_{k=1}^{\infty} k\left|b_{k}\right|^{2} .
$$

Bemerkungen. 1. Eine zu (22) ähnliche Ungleichung für die Klasse $\Sigma(\kappa)$ (mit $0<\kappa<1$ ) ergibt die Herleitung in [11], S. 291, wenn man diese in der 6. Zeile von oben stoppt. Dann haben wir nämlich eine Ungleichung, die aus (22) bei Ersetzung von $\leq$ durch $\geq$ entsteht (und hieraus folgen in [11], S. 291, die Grunskyschen Koeffizientenbedingungen für die Klasse $\Sigma(\kappa)$ ).

2. Zur Frage des Gleichheitszeichens in (22): Zwar kann man (analog wie in der Klasse $\Sigma(\kappa)$ ) aus der Annahme des Gleichheitszeichens in (22) Bedingungen für die betreffenden Abbildungen $g(z)$ herleiten, aber es bleibt die Frage, ob diese Bedingungen wirklich von konkreten Abbildungen in der Klasse $\widetilde{\Sigma_{s}}(\kappa)\left(\right.$ mit $\left.1<\kappa_{s}\right)$ erfüllt werden. In der Klasse $\Sigma(\kappa)($ mit $0<\kappa<1)$ kann man dieses Problem bekanntlich dadurch umgehen, daß man das betreffende Extremalproblem mit einer Variationsmethode studiert. Aus Kompaktheitsgründen besitzt dann das Extremalproblem Lösungen, und die betreffenden Abbildungen müssen gewisse notwendige Bedingungen erfüllen, die ebenfalls zur (22) entsprechenden Ungleichung in der Klasse $\Sigma(\kappa)$ mit $0<\kappa<1$ führen. So konnte in [7] die entsprechende scharfe Ungleichung gewonnen werden. Diese Methode versagt hier leider vollständig, da in der Klasse $\widetilde{\Sigma_{s}}(\kappa)$ (noch) keine analogen Variationsmethoden zur Verfügung stehen. Damit bleibt hier offen, ob in (22) das Gleichheitszeichen wirklich stehen kann.

3. Ungleichung (22) kann man auch für die größere Klasse $\widetilde{\Sigma}(\kappa)$ herleiten, muß aber in Kauf nehmen, daß die unendliche Reihe in (22) gar nicht konvergiert (vgl. schon den genannten Spezialfall $m=1$ ), was das Resultat wie beim Flächensatz (5) gegenstandslos macht.

5. Zu Einzelkoeffizienten. Es scheint, daß - im Gegensatz zur Klasse $\Sigma(\kappa)$ - in der Klasse $\widetilde{\Sigma_{s}}(\kappa)$ Abschätzungen für Einzelkoeffizienten $b_{k}$ (und auch Abschätzungen für das eigentliche Grunskysche Funktional entsprechend der ersten Summe in (22)) nicht möglich sind. (Analogie: Für die Punkte des Inneren der Einheitskugel im z.B. 3-dimensionalen euklidischen Raum gelten natürlich Abschätzungen für die 3 Koordinaten, nicht aber für die Punkte des Äußeren.) Erhellend zu dieser Frage ist immerhin die Betrachtung der folgenden Beispiele. 
1. Bei den Abbildungen $g(z)$ der Klasse $\widetilde{\Sigma}(\kappa)$ ist zumindest für $1<\kappa<3$ das Verschwinden des Koeffizienten $b_{1}$ in (1) möglich. Das zeigen bei hinreichend großen $p$ die Abbildungen

$$
g(z)= \begin{cases}z+\frac{p}{z^{2}} & \text { für }|z| \geq 1 \\ z+p \bar{z} \frac{|z|}{z} & \text { für }|z| \leq 1 .\end{cases}
$$

Man errechnet nämlich wegen $|z|=\sqrt{z \bar{z}}$

$$
g_{z}=1-p \frac{1}{2} \bar{z}^{3 / 2} z^{-3 / 2}, \quad g_{\bar{z}}=p \frac{3}{2} \frac{\bar{z}^{1 / 2}}{z^{1 / 2}},
$$

so daß (3) erfüllt ist, also $g(z) \in \widetilde{\Sigma}(\kappa)$ gilt, falls

$$
\kappa\left(1+\frac{1}{2} p\right) \leq \frac{3}{2} p
$$

ist. Das ist tatsächlich zu festem $\kappa<3$ gewährleistet für alle hinreichend großen $p$.

Diese Abbildungen (24) sind allerdings für hinreichend große $p$ nicht schlicht für $|z|<1$. Man vgl. z.B. die Abbildung auf den Kreisen $|z|=r$. Für $z=r e^{i \varphi}$ gilt nämlich

$$
g(z)=r\left(e^{i \varphi}+p e^{-2 i \varphi}\right) .
$$

Offen bleibt die Frage, ob auch $b_{1}=0$ möglich ist bei einer Abbildung $g(z)$ der Klasse $\widetilde{\Sigma}(\kappa)$ bei $\kappa \geq 3$. Und es bleibt offen, ob es solche Abbildungen $g(z)$ gibt, die sogar für $\mid z<1$ schlicht sind, also zur Klasse $\widetilde{\Sigma}_{s}(\kappa)$ gehören. In diesem Zusammenhange eine Berichtigung $z u$ [8]: Die dort auf S. 262 angegebene Funktion $\sqrt{w^{*}\left(z^{2}\right)}$ ist untauglich, um zu zeigen, daß $a_{1}=0$ (entsprechend $b_{1}=0$ in vorliegender Mitteilung) sein kann, z.B. weil diese Funktion in Umgebung der 4 Stellen $\sqrt[4]{-p}$ (für $p>1$ ) gar nicht eindeutig ist, da $w^{*}\left(z^{2}\right)$ dort Nullstellen erster Ordnung besitzt.

2. Die Abbildungen (24) zeigen, daß $\left|b_{1}\right|$ in der Klasse $\widetilde{\Sigma}(\kappa)$ für jedes $\kappa>1$ beliebig groß werden kann. Ferner zeigt (24), daß hier auch $\left|b_{2}\right|$ zumindest für $1<\kappa<3$ beliebig groß werden kann. Allgemein zeigt bei $n \geq 2$ die Abbildung

$$
g(z)= \begin{cases}z+\frac{p}{z^{n}} & \text { für }|z| \geq 1 \\ z+p \bar{z}\left(\frac{|z|}{z}\right)^{n-1} & \text { für }|z| \leq 1\end{cases}
$$

daß $\left|b_{n}\right|$ zumindest für

$$
1<\kappa<\frac{n+1}{n-1}
$$


beliebig groß werden kann. Denn für (26) ist (3) erfüllt bei

$$
\kappa\left(1+p \frac{n-1}{2}\right) \leq p \frac{n+1}{2} \text {. }
$$

\section{LITERATUR}

[1] Ahlfors, L. V., A remark on schlicht functions with quasiconformal extensions, Symposium on Complex Analysis, Canterbury 1973, London Math. Soc. Lect. Note Series 12, Cambridge Univ. Press, Cambridge, 1974, 7-10.

[2] Hoy, E., Flächensätze für quasikonform fortsetzbare Abbildungen, Z. Anal. Anwendungen 3 (1984), no. 1, 19-31.

[3] Hoy, E., Flächensätze für quasikonform fortsetzbare Abbildungen mit ortsabhängiger Dilatationsbeschränkung, Math. Nachr. 121 (1985), 147-161.

[4] Hoy, E., Flächensätze in Klassen von quasikonform fortsetzbaren konformen Abbildungen, Math. Nachr. 128 (1986), 265-285.

[5] Hoy, E., Einige Bemerkungen zu Flächensätzen für quasikonform fortsetzbare Abbildungen, Math. Nachr. 131 (1987), 89-99.

[6] Hoy, E., Area theorems and Fredholm eigenvalues, Ann. Acad. Sci. Fenn. Ser. A. I. Math. 14 (1989), 137-148.

[7] Kühnau, R., Verzerrungssätze und Koeffizientenbedingungen vom GRUNSKYschen Typ für quasikonforme Abbildungen, Math. Nachr. 48 (1971), 77-105.

[8] Kühnau, R., Eine Klasse nichtschlichter konformer Abbildungen mit einer schlichten quasikonformen Fortsetzung, Math. Nachr. 59 (1974), 261-263.

[9] Kühnau, R., Complete anti-analytic continuation of analytic functions, Georgian Math. J. 17 (2010), 305-345.

[10] Lehto, O., Schlicht functions with a quasiconformal extension, Ann. Acad. Sci. Fenn. Ser. A. I. Math. no. 500 (1971), 1-10.

[11] Pommerenke, Ch., Univalent Functions, Vandenhoeck \& Ruprecht, Göttingen, 1975.

Reiner Kühnau

FB Mathematik der Martin-Luther-Universität Halle-Wittenberg

D-06099 Halle/Saale

Deutschland

e-mail: kuehnau@mathematik.uni-halle.de

Received April 26, 2011 